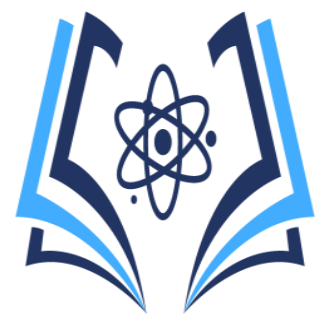

\title{
Factores de riesgos que provocan la caries dental en los afiliados de la Unidad de Salud "La Piñuela"
}

\section{Risk factors that cause dental caries in the members of the "La Piñuela" Health Unit}

\section{Riesgos que provocan la caries dental}

\author{
Gema Stefany Barreiro Mendoza. MSc ${ }^{(1)}$ \\ Roberth William Zambrano De La Torre. MSc ${ }^{(2)}$ \\ ${ }^{1}$ Instituto Ecuatoriano de Seguridad Social. Manabí. Ecuador. gema.barreiro@iess.gob.ec, Código Orcid: \\ https://orcid.org/0000-0001-9661-9761 \\ ${ }^{2}$ Instituto Superior Universitario Portoviejo. Manabí. Ecuador. zroberthwilliam@itsup.edu.ec, Código Orcid: \\ https://orcid.org/0000-0001-5450-5037
}

Contacto: gema.barreiro@iess.gob.ec

Recibido: 12-08-2021

Aprobado: 10-09-2021

\section{Resumen}

La caries dental es una sucesión anómala compleja, de causa contagiosa y traspasable, que altera las estructuras de las piezas dentarias, además se ven influenciados con afectación durante la alimentación y la nutrición; asimismo, altera la forma psicológica como social de quienes lo padecen. La prevalencia de caries a nivel mundial, según los datos de la Organización Mundial de la Salud, es de casi el 100\% en personas adultas. Esta investigación analiza la prevalencia de caries dental e identifica los factores de riesgo para su aparición y distribución en los afiliados al Seguro Social Campesino que recibieron atención odontológica durante los meses de septiembre a noviembre de 2020, en la Unidad de Salud "La Piñuela", ubicado en el cantón Tosagua, en Ecuador. Se diseñó, un estudio descriptivo de corte transversal, en el que, a partir del estudio de la valoración de los índices de dientes cariados, obturados y perdidos por caries, se determinó el índice de caries dental que se presenta en los afiliados; y, a partir métodos observacionales $\mathrm{y}$ analíticos se identificaron los factores de riesgo que originaron la aparición de caries. Los resultados de esta investigación servirán para establecer protocolos en la prevención de caries dental, teniendo en cuenta su distribución y aparición a partir de su asociación con factores socioculturales, económicos, del ambiente y de comportamiento.

Palabras clave: Caries dental, factores de riesgo, higiene dental.

\section{Abstract}

Tooth decay is a complex pathological process of in fectious and communicable origin that affecs tooth $\mathrm{s}$ tructures. This process affects basic vital functions such as food and nutrition and alters other more complex ones that psychosocially influence people's relationship lives. Data from the World Health Organization indicate that the worldwide prevalence of tooth decay is almost $100 \%$ in adults. This research aims to analyze the prevalence of dental caries and identify the risk factors for its appearance and distribution in the members who received dental care during the months of September to November 
2020, at the "La Piñuela" Health Unit, located in the canton Tosagua, in Ecuador. For this purpose, a descriptive cross-sectional study was designed, in which a study of the assessment of the COPD indices (decayed, filled and lost teeth due to caries) was determined the index of dental caries that occurs in the affiliates; and, from observational and analytical methods, the risk factors that originated the appearance of caries were identified. The results of this research will allow us to establish protocols in the prevention of dental caries, taking into account its distribution and appearance from its association with sociocultural, economic, environmental and behavioral factors.

Keywords: Dental caries, risk factors, dental hygiene Introducción

El daño de estructuras dentarias es un proceso que puede iniciar en edad temprana ante la presencia adversa de condiciones higiénicas, alimentarias, ambientales y económicas (1). La caries dental afecta aproximadamente entre el $95 \%$ al $99 \%$ de la población mundial, lo que la cataloga como la que origina la pérdida de estructuras dentarias, ya que nueve personas de cada diez padecen de caries dental o enfermedades asociadas a ella (2).

La caries dental se caracteriza por realizar un proceso que actúa desmineralizando los tejidos duros de los dientes, su complejidad radica que al ser un proceso infeccioso puede transmitirse fácilmente y afectar a todas las estructuras dentarias (3); y, si no es tratado a tiempo, conduce a producir cavidades profundas con alteraciones irreversibles de su tejido dentinopulpar (4).

La Organización Mundial de la Salud (OMS) define la caries dental como un proceso patológico localizado que se inicia tras la erupción dentaria y produce reblandecimiento del tejido duro del diente, evolucionando hasta la formación de una cavidad, y se la atribuye principalmente a la bacteria Streptococcus mutans (5). También se define como
Periodo. Julio - Diciembre 2021 Vol. 5, Nro. 2, Publicado: 2021-12-31 un proceso patológico caracterizado por la destrucción localizada de los tejidos duros susceptibles del diente que ocurre por la interacción de numerosos factores de riesgo $y$ factores protectores, tanto a nivel de la cavidad bucal como a nivel individual y social (6).

La caries activa produce una destrucción que avanza muy rápido hacia la estructura dental por la presencia de un ambiente ácido causado por la presencia de microorganismos cariogénicos y alimentado por la ingesta de fermentos azucarados (7). Entonces, para que se presente la caries dental tienen que producirse ácidos durante un determinado tiempo, y la microbiota y un sustrato bacteriano deben interactuar con el esmalte. El tiempo que conlleve este proceso dependerá de la estructura química del esmalte y por lo tanto de su resistencia a la desmineralización por los ácidos bacterianos (8).

El desarrollo de la desmineralización es provocado por múltiples factores, como el tipo de dieta, apiñamientos dentarios, además se relacionan con enfermedades sistémicas, y el tiempo que permanecen estos elementos en la cavidad oral junto con una deficiente higiene bucal (9). Se evidencian, entonces, tres factores esenciales, además del tiempo: huésped (saliva y dientes), microorganismos (microflora) y dieta (sustrato) (9). El huésped va a proveer un ambiente favorable o desfavorable para la formación de la caries, ya sea por su nivel de higiene o por predisposición genética (10).

La caries dental interfiere en la vida diaria en acciones básicas del ser humano como la alimentación y la nutrición, y en situaciones más complejas provoca daños psicológicos en la vida de las personas que las presentan (11); sin embargo, puede ser reversible si el proceso en su etapa inicial de desmineralización dental se detecta a tiempo y se eliminan las estructuras del biofilm microbiano (8), pero si no ocurre un proceso de remineralización se 
Periodo. Julio - Diciembre 2021

Vol. 5, Nro. 2, Publicado: 2021-12-31

producirá la pérdida de la estructura dental en cualquiera de sus superficies (8).

La OMS (12) en su plan de acción para la promoción y prevención de la salud bucal, recomienda la incorporación de estrategias y enfoques comparables con los del tratamiento de enfermedades crónicas. Esta preocupación se abordó en la Asamblea General de las Naciones Unidas sobre la prevención y control de enfermedades no transmisibles que estableció aumentar la atención de los crecientes niveles de este tipo de enfermedades como la salud dental (13).

Cerón Bastidas (10) explica que entre las opciones de los métodos diagnósticos se encuentran el sistema ICDAS (International Caries Detection and Assessment System) y NYVAD (Sistema creado por el autor Nyvad), la exploración clínica, la inspección visual, la exploración táctil con sonda, radiografía digital, transiluminación (FOTI), el método de conductibilidad eléctrica (ECM), y fluorescencia entre otros (10).

Uno de los índices epidemiológicos de fácil relevamiento es el CPOD, (dientes cariados, perdidos, obturados en dentición permanente), que fue desarrollado por Klein, Palmer y Knutson (1938) durante un estudio sobre el estado y la necesidad tratamiento dental en niños asistentes a escuelas primarias en Hagerstown, en Maryland, EE.UU. (14). El índice CPOD mide la presencia de dientes con caries en la dentición permanente o del adulto y hace referencia si el diente esta restaurado o necesita realizarse extracción dentaria (15).

Vélez Vásquez et al. (16) explican que desde 1938, algunos países ponen en práctica el índice CPOD para determinar y controlar su desarrollo en la prevención de caries. Precisamente, la OMS da a conocer constantemente los resultados promedios nacionales de CPOD de todos los países que organizan sus políticas de salud con esta institución, y crean esquemas epidemiológicos con este indicador (16).
González Ferrer et al. (17) sostienen que los valores para identificar el riesgo de contraer caries ajustados a contextos de aplicación específicos permiten focalizar intervenciones preventivas en las personas, y se pueden desarrollar a partir de diferentes procedimientos (17). Los factores de riesgos de presentar caries son mediante observación clínica que pueden presentar a partir de diferentes sistemas de detección y funcionan para decidir el nivel de riesgo, que puede ser alto, medio o bajo (18):

- Los pacientes de bajo riesgo no requieren de tratamientos específicos; sin embargo, hay que brindarles educación bucal y monitoreo anual.

- Los pacientes de riesgo medio presentan elementos que justifican un tratamiento que incluya medidas correctivas o preventivas, el cambio de alimentación o el uso de sustancias que contengan flúor.

- Los pacientes de alto riesgo requieren un tratamiento mediante procedimiento adecuados a cada caso, alimentación balanceada, aumento de consumo de líquidos, medidas bacteriológicas, aplicación tópica de fluoruros (19).

Algunos de los indicadores de riesgo sugeridos por Andrade Páez y De La Cruz Cardoso (18) son las lesiones en el esmalte dental que se presentan en etapas iniciales como una mancha blanca en superficies pudiendo ser en superficies lisas o las restauraciones colocadas anteriormente por antecedente de caries. Por otro lado, sugieren como los factores de riesgo, el caso de las bacterias cariogénicas, poca producción de saliva, estos se mencionan como variables que pueden provocar las lesiones de caries (18).

En los tiempos actuales como medida preventiva se realiza la aplicación de flúor por aplicación tópica o por vía sistémica. Como explican Gumila Jardines et al. (20), las medidas de prevención contra la caries 
Periodo. Julio - Diciembre 2021

Vol. 5, Nro. 2, Publicado: 2021-12-31

dental pueden ser aplicados de forma individual, familiar y en toda una comunidad. En esta situación, para que se puedan realizar, deben existir dos condiciones: 1) la prevención sea una necesidad y 2) la prevención debe ser tolerada por toda la población (20).

Esto accede a declarar que la salud integral y la vida se ven afectadas como resultado de la caries dental (11). Entonces, al ser una afección que permite hacer un diagnóstico precoz (21), se deben aplicar protocolos de prevención para mantener al paciente libre de caries (22), pero si ya presenta la enfermedad se debe evitar su avance a través de su identificación por medio de los índices de diagnóstico para detectar y cuantificar las afectaciones por caries (23), y el tratamiento necesario en quienes requieran aplicación de procedimientos de prevención.

La literatura revisada ha permitido analizar a la caries dental desde la visión integral, como desde las consecuencias que tiene esta enfermedad en la salud pública. Como caso particular, la Unidad de Salud "La Piñuela" es parte de la red de salud pública ecuatoriana, el Instituto Ecuatoriano de Seguridad Social (IESS), que atiende a los afiliados del Seguro Social Campesino y está ubicada en una zona rural de la provincia de Manabí. El centro de salud de atención integral más cercano está ubicado a 76 kilómetros de la zona, por lo que en esta Unidad de Salud se ofrece una atención primaria, y de ser necesario se derivan los casos a los hospitales que forman parte de la red de hospitales del IESS. Cuenta con 2 médicos generales, 1 odontólogo y 2 enfermeras, y atiende entre 10 a 50 pacientes diariamente.

La pertinencia de este estudio radica en que las Unidades de Salud ubicadas en zonas rurales no han sido objeto de investigación previa, de manera que un estudio se hace necesario para realizar intervenciones preventivas de salud bucal. Es importante, entonces, porque se necesita poder reconocer que la caries dental ocupa un lugar dentro de los problemas de salud de los afiliados y la gran variedad de factores de riesgos que intervienen en él, al identificarse, pueden permitir realizar los protocolos necesarios para el beneficio de éstos.

\section{Materiales y métodos}

Se realizó un estudio observacional descriptivo de corte transversal en 83 pacientes adultos afiliados al Instituto Ecuatoriano de Seguridad Social Campesino que recibieron atención en el Área de Odontología de la Unidad de Salud "La Piñuela", afectados por caries dental, de una población de 478 pacientes entre niños y adultos. Se determinó la muestra a partir de la identificación de tres grupos de pacientes: el grupo A corresponde a pacientes hombres y mujeres de entre 20 a 39 años; el grupo B corresponde a pacientes hombres y mujeres de entre 40 a 64 años; el grupo C corresponde a pacientes de 65 años en adelante.

Se emplearon las historias clínicas de salud bucal ejecutadas por el Odontólogo en las que utilizó la unidad dental y el set de examinación que incluye espejo, pinza, explorador y luz artificial. Para la determinación de la muestra a analizar, se seleccionaron las historias clínicas de los pacientes pertenecientes al rango etario seleccionado para la investigación, y se realizó un muestreo aleatorio donde el nivel de confianza es del $95 \%$ y el margen de error es del 5\%, lo cual dio como resultado el tamaño de la población investigada un total de 104 pacientes, y la muestra un total de 83 pacientes.

Para determinar la severidad de caries dental, se obtuvo la valoración por medio de los índices COPD que tienen como objetivo valorar el desarrollo carioso en su etapa inicial, además de su severidad y fases de acción (24).

Para determinar los factores de riesgo, se recabó información de los pacientes identificados dentro del rango etario seleccionado para esta investigación a partir de una encuesta y una ficha de indicadores de la enfermedad, en la que se incluyeron variables 
Periodo. Julio - Diciembre 2021

sociodemográficas como la edad, la actividad laboral, Vol. 5, Nro. 2, Publicado: 2021-12-31 los antecedentes previos de caries, entre otras variables.

\section{Resultados}

El presente estudio se realizó durante el periodo del 15 de septiembre al 15 de noviembre de 2020, a partir de la elaboración y posterior análisis de las historias clínicas de 83 pacientes afiliados al Seguro Social Campesino, hombres y mujeres de entre 20 y más de 65 años, que solicitaron atención médica odontológica.

El total de pacientes odontológicos atendidos en la Unidad de Salud "La Piñuela", durante el periodo de estudio, se realizó una preclasificación de las historias clínicas para excluir a los pacientes odontológicos que no pertenecían al rango etario clínicas (033), del total de pacientes odontológicos atendidos en el periodo seleccionado, y se dividieron en tres grupos. A cada 033 se le adjuntó una ficha y una encuesta que se llenó durante la consulta.

La división en grupos etarios permitió identificar las prevalencias de distintos rangos como los motivos de consulta, y los indicadores de salud bucal. También permitió identificar las formas de cuidado $\mathrm{y}$ antecedentes de caries, a partir de las encuestas realizadas. A continuación, se presentan los resultados del análisis de las historias clínicas 033 de los pacientes atendidos durante el periodo de estudio, dividido en hombres y mujeres, y los diagnosticados con caries, y con otro tipo de diagnóstico.

Tabla 1. División de grupos según rango etario y DX de caries

\begin{tabular}{lccccc}
\hline Grupo & Total & Hombres & Mujeres & Dx caries & Otro dx \\
\hline A (20 a 39 años) & 43 & 7 & 36 & 39 & 4 \\
\hline B (40 a 65 años) & 32 & 10 & 22 & 27 & 5 \\
\hline C (65 años en adelante) & 8 & 4 & 4 & 8 & -
\end{tabular}

Fuente: Autora de la investigación

Se identificó la prevalencia de pacientes con diagnóstico de caries en el grupo A; así como mayor cantidad de pacientes de sexo femenino en los grupos A y B. En el caso del grupo C, se evidencia la misma cantidad en sexo femenino y masculino.

Entre los motivos de consulta prevalece la limpieza dental en todos los grupos. Se evidencia, sin embargo, que los diagnósticos odontológicos tienen en un $73 \%$ de las atenciones caries dental, en un $18 \%$ extracción y en un $9 \%$ otros diagnósticos, como gingivitis o pulpitis.
En todos los grupos prevalece la no existencia de antecedentes personales y familiares de enfermedad. Dentro de la historia clínica se detallan alergia antibiótica o a anestesia, hemorragias, VIH/SIDA, tuberculosis, asma, diabetes, hipertensión, enfermedades cardiacas y otros. Se debe tener en cuenta que la mayor cantidad de pacientes atendidos están dentro del rango etario 20 a 39 años. En el Gráfico 1, se evidencia la presencia de hipertensión y diabetes como los antecedentes que registraron los pacientes atendidos.

\section{Antecedentes personales y familiares}




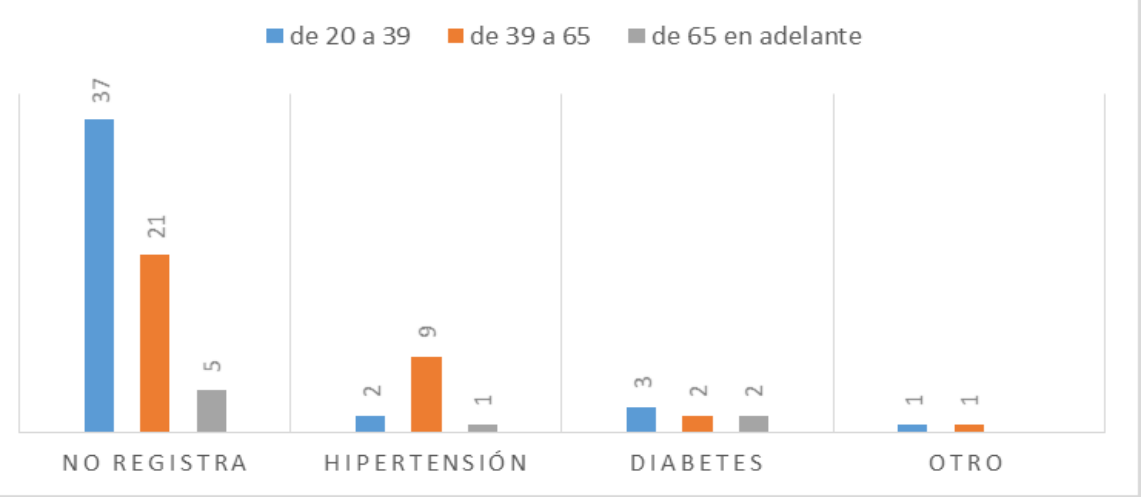

Gráfico 1 Resultado de antecedentes personales y familiares en Historia Clínica 033.

Con respecto de los resultados de las historias clinicas al Odontograma de cada paciente, se realizó una sumatoria en los índices CPOD, separados por rangos. No se realizó distinción entre el detalle del tratamiento realizado o la patologia actual, movilidad y/o recesión, ya que el interes de la investigación es identificar una existencia general de la caries dental en los pacientes.

\section{Índice COPD-CEO}

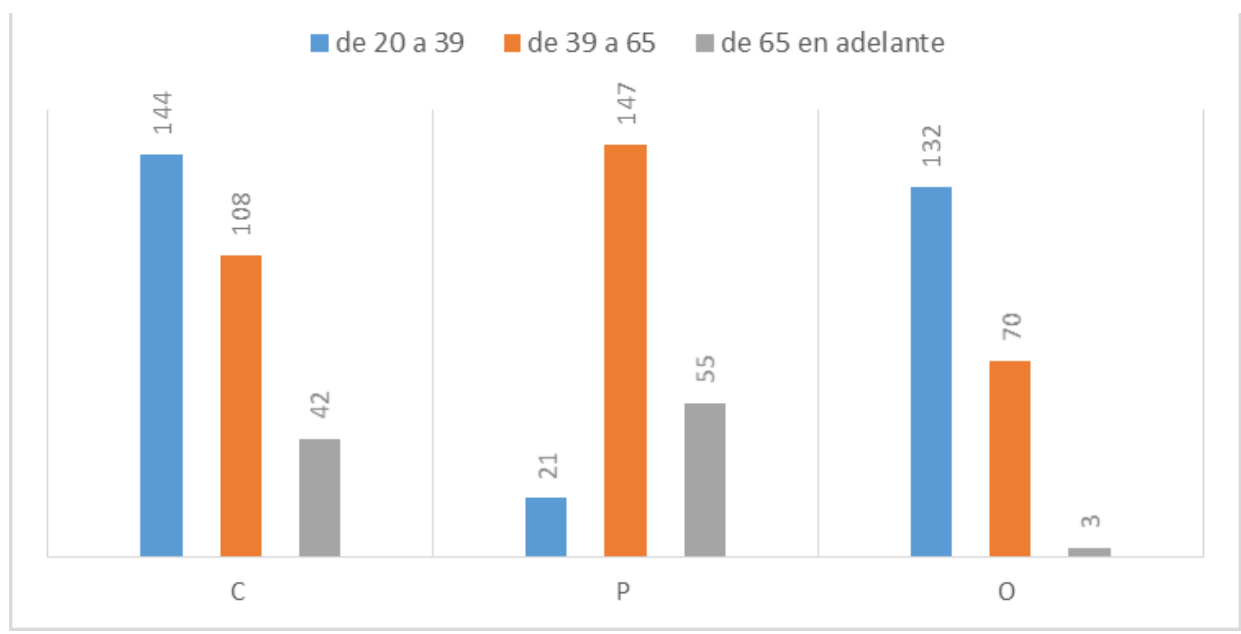

\section{Gráfico 2. Índice COPD.}

Por su parte, como se presenta en el Gráfico 3, en el grupo A que asistió a la consulta por motivo de limpieza bucal se refleja mayor presencia de Placa a
Los resultados del Gráfico 2 reflejaron que en el grupo A existe mayor prevalencia de dientes cariados y obturados, a diferencia del grupo B donde los dientes perdidos tienes mayor prevalencia que los cariados. En el caso del grupo $\mathrm{C}$ se evidencia una cantidad paralela entre dientes cariados y perdidos. nivel del tercio gingival. En el grupo B, se evidencian otros factores como el cálculo o la gingivitis.

\section{Higiene oral simplificada}




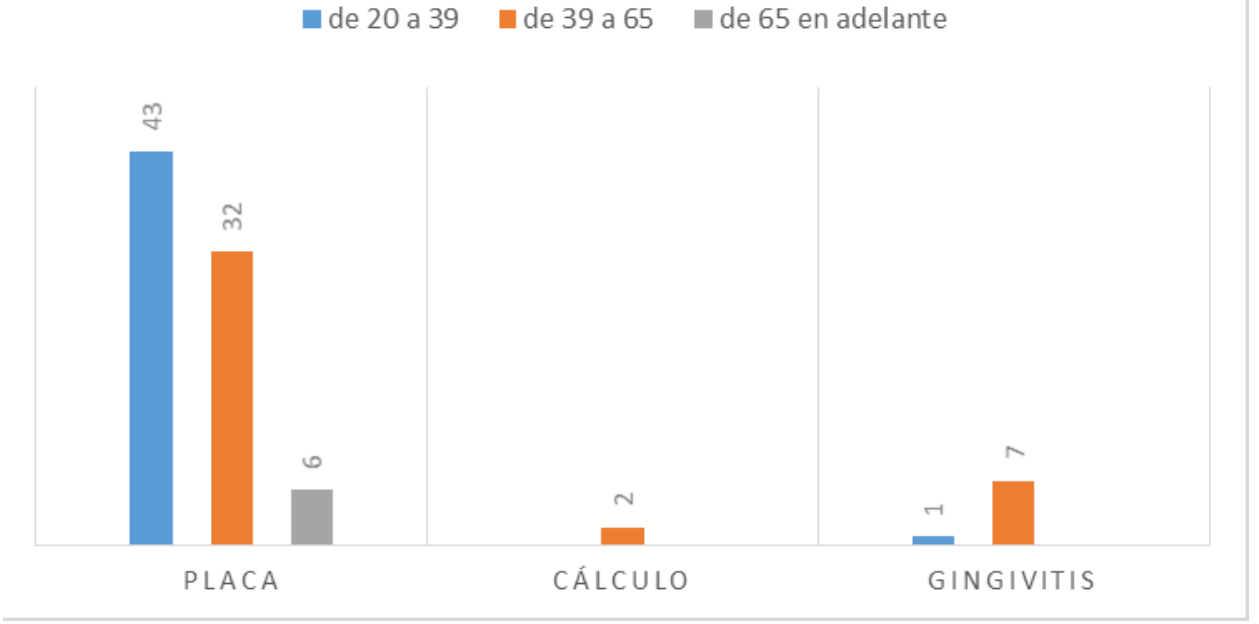

Gráfico ¡Error! No hay texto con el estilo especificado en el documento. Resultado de higiene oral

\section{simplificada.}

El resultado del análisis de las historias clínicas arroja que el $90 \%$ de pacientes diagnosticados con caries, recibieron profilaxis y educación para la salud. Al 9\% de los pacientes se les realizó extracciones y al 1\%, restauraciones. La presencia de placa en todos los grupos de la investigación se refleja en la necesidad de realizar profilaxis $\mathrm{y} /$ restauraciones.

\section{Factores de riesgo}

Se establecieron dos herramientas de recolección de datos para identificar los factores de riesgo a caries existentes: una encuesta de 16 preguntas cerradas y opción múltiple, que se realizó a cada paciente que recibió atención odontológica. Se realizó también una ficha con indicadores de factores que fue completado por el profesional a partir de la entrevista a cada paciente.

De las herramientas de recolección de datos mencionadas se identificó que el $61 \%$ de pacientes del grupo A, el 52\% del grupo B y el 50\% del grupo C están relacionados con actividades agrícolas. Por su parte, el 36\% del total de pacientes tienen instrucción primaria, y el 29\% tienen instrucción secundaria. El 15\% de los pacientes realiza estudios universitarios y pertenecen al grupo A, y el $20 \%$ no tiene estudios realizados. Se tuvo en cuenta que la
Unidad de Salud se encuentra ubicada en un área rural de la provincia.

Con respecto de la frecuencia de visita al Odontólogo, prevalece en todos los grupos la vista una vez al año, y sobre la frecuencia de cepillado y uso de enjuague o hilo dental se evidencia que el grupo A asegura cepillarse 2 veces al día, mientras que los grupos B y C lo realizan 1 vez al día, mientras que el $72 \%$ del total de pacientes respondieron no usar nunca o casi nunca el enjuague y el hilo dental. Con respecto de los antecedentes de caries, el $93 \%$ del total de pacientes afirmó tener o haber tenido caries. La mayor cantidad de pacientes con antecedentes de caries recae en el grupo 1. En cuanto a la temporalidad, 42 de los 83 pacientes analizados sostuvieron que fueron diagnosticados con caries hace un año. Es importe destacar que los 6 pacientes que sostuvieron nunca haber tenido caries, fueron diagnosticados con esta afección durante la consulta odontológica que sirvió para realizar este estudio. El $63 \%$ de total de pacientes atendidos señaló consumir alimentos azucarados más de dos veces al día. El grupo con mayor consumo de esta clase de alimentos es el 1. Por su parte, el $81 \%$ reportó tener sensación de boca seca.

Tabla 2. Indicadores de la enfermedad 


\begin{tabular}{|c|c|c|c|c|c|c|}
\hline & $\mathbf{H}$ & $\mathbf{M}$ & $\mathbf{H}$ & $\mathbf{M}$ & $\mathbf{H}$ & $\mathbf{M}$ \\
\hline $\begin{array}{l}\text { Presencia de lesiones de caries } \\
\text { cavitadas }\end{array}$ & 5 & 28 & 9 & 20 & 4 & 3 \\
\hline $\begin{array}{l}\text { Imágenes radiolúcidas compatibles } \\
\text { con pérdidas de sustancia } \\
\text { calcificada }\end{array}$ & 6 & 29 & 8 & 21 & 4 & 4 \\
\hline $\begin{array}{l}\text { Presencia de lesiones iniciales de } \\
\text { caries (mancha blanca) }\end{array}$ & 6 & 30 & 10 & 20 & 4 & 4 \\
\hline $\begin{array}{l}\text { Restauraciones en los últimos tres } \\
\text { años }\end{array}$ & 7 & 30 & 10 & 21 & 4 & 3 \\
\hline
\end{tabular}

\section{Fuente: Autora de la investigación}

Se evidencian los indicadores establecidos para determinar la enfermedad en los pacientes atendidos durante el periodo de estudio. Esta ficha permitió cotejar la información brindada por el paciente en la encuesta, a partir de la observación del odontólogo que realizó la consulta respectiva. De esta ficha se evidenció que en los tres grupos se puede existe un mayor porcentaje de presencia de indicadores como lesiones iniciales de caries, restauraciones realizadas anteriormente y caries cavitadas. Asimismo, los resultados recabados tanto en las fichas clínicas como en las encuestas evidencian un riesgo a caries de 93\%, debido a la presencia de factores biológicos predisponentes, como la placa dental visible en el $89 \%$ de los casos atendidos, así como el alto consumo de alimentos azucarados y flujo salival reducido. Los factores protectores como la poca frecuencia del cepillado, la ausencia de uso de enjuague bucal, hilo dental o consumo de xilitol, también contribuyen con la elevada presencia de riesgo a caries encontradas en el presente trabajo.

\section{Discusión}

La literatura estudiada coincide con el presente trabajo al identificar a la caries dental como una de las enfermedades de origen infeccioso de mayor prevalencia y uno de los principales problemas de salud pública a nivel mundial. Gonzáles Ferrer (17) sostiene que en el caso de las afecciones prevenibles como la caries dental, el correcto reconocimiento del grupo de riesgo es lo más importante, ya que ayuda a planificar estrategias de prevención de la enfermedad sin gastos innecesarios de recursos. Esto permitirá, como expresan Toledo, Rodríguez y Ramos (25), brindar a la población elementos que le inviten a crear una obligación con respecto a su salud oral. Esto puede evidenciarse en los resultados arrojados en el presente estudio, teniendo en cuenta que el principal grupo de riesgo se encuentra en adultos de entre $20 \mathrm{y}$ 39 años.

Es importante reconocer los factores que conllevan a la aparición de esta enfermedad, porque pueden indicar algunas formas de actuación que ayuden a evitar la intensidad de la enfermedad (17). La técnica inadecuada del cepillado dental, por ejemplo, contribuye a la aparición de caries dental de forma más frecuente, lo que se destaca en los resultados de las encuestan en las que los pacientes de 20 a 39 años señalan cepillarse diariamente, pero sin embargo es el grupo con mayor índice de placa. No obstante, este resultado puede cambiar de forma favorable con una 
adecuada estrategia de educación en lo referente a la salud bucal.

Siguiendo a Cerón Bastidas (10), en la actualidad hay unos 29 sistemas para identificar y determinar la caries dental, lo que permite un diagnóstico oportuno y la identificación de aquellos pacientes que necesitan trabajo intenso de medidas preventivas. Precisamente, diversos autores coinciden en que la prevención es la herramienta menos costosa y más eficaz para evitar la enfermedad de la caries dental, y la evaluación mediante observación siguen considerándose los más precisos, específicos y sensibles en la detección de esta enfermedad.

Precisamente, Vélez Vásquez et al. (16) explican que por referencia de la OMS se determina la salud bucal mediante el índice CPOD. Este índice enumera las piezas dentarias cariadas en el momento de la atención odontológica, las que en tiempos pasados fueron cariadas y se le realizó obturación y las piezas dentarias pérdidas a causa de la caries dental. Este valor de experiencia de caries permite predecir la cantidad de caries que se tendrá en un tiempo considerado más adelante, con una sensibilidad del $52 \%$ y una especificidad del $92 \%$ (16). El uso de este índice aparece en la Historia Clínica elaborada por un subsistema de salud pública, como es el caso del Instituto Ecuatoriano de Seguridad Social, y coincide con el sistema de salud regente en el país, es el caso del Ministerio de Salud Pública.

Por su parte, Andrade Páez y De la Cruz Cardoso (18) sostienen que la evaluación del riesgo de caries permite un pronóstico que brinde una programación tanto de los tratamientos preventivos como de los curativos. Cantillo, Elías Sierras y Rodríguez (26) sugieren que en el ámbito comunitario, reconocer el riesgo de caries da la oportunidad de determinar planes de prevención direccionados a pacientes con un elevado riesgo.

La revaloración del riesgo de caries del paciente debe ocurrir entre el primer trimestre a un año posterior a
Vol. 5, Nro. 2, Publicado: 2021-12-31

la primera valoración (27), en referencia con el porcentaje de riesgo obtenido del paciente. Asimismo, algunos de los métodos de los pronósticos de caries toman en cuenta entre ocho y 18 factores tanto de riesgo, como de prevención (18). En general, estos procedimientos tratan de realizar un modelo de revelación que sea sencillo, preciso, rápido y económico.

Los factores de riesgos que pueden producir las caries son diversos y compuestos, entre los cuales se tienen los de carácter biológico, el estilo de alimentación y los hábitos de higiene y limpieza bucal (28). Dichos elementos permiten analizar a esta enfermedad como un tema de salud pública debido a la elevada incidencia y prevalencia, especialmente en los países en vías de desarrollo.

En estos tiempos se ha demostrado un alto interés en el estudio del efecto de diferentes procedimientos preventivos no operatorios de la caries dental (29). La inspección de estos fenómenos solicita un método de diagnóstico que revele el aspecto dinámico de la caries en todas los procesos de desarrollo de la lesión (30). Es por esto que las actuales condiciones de detección y nuevos procedimientos de tratamiento sugieren una descripción de la caries que acceda a instaurar una diferencia entre el proceso de la enfermedad de caries y la lesión de caries, centrándose en la necesidad de manejar la enfermedad y de remineralizar la lesión (19).

En suma, se coincide con trabajos anteriores a la elaboración de este estudio en que la esencia multifactorial de la caries dental puede ser el resultado de condiciones dispares donde distintos estilos y modos de vida (31), condiciones socioeconómicas adversas y diversos modelos de atención de la salud, pueden transformarse, en los factores determinantes de un estado de salud bucal determinado (32), por lo que debe tratarse de una manera integral, valorizando la prevención de la enfermedad. 


\section{Conclusiones}

El estudio realizado determinó el índice de caries dental a partir de la valoración COPD realizada en la historia clínica de cada paciente atendido en el área odontológica de la en la Unidad de Salud "La Piñuela", donde se evidencia la alta prevalencia de caries en el grupo A. Dato a destacar pues las encuestas arrojaron que este grupo detalló una frecuencia mayor de cepillado y de uso de enjuague/hilo dental (dos veces al día), pero también reflejó una mayor cantidad de consumo de alimentos azucarados (dos veces). Por su parte, al ser el grupo A, el que tiene un mayor nivel de instrucción, se le puede atribuir que el número de dientes perdidos y obturados es menor, debido a que van a la consulta odontológica con mayor frecuencia ( 2 veces al año). Se indagó en los factores de riesgo que originaron la aparición de caries y se pudo evidenciar el consumo de alimentos azucarados y la prevalencia al cepillarse los dientes una vez al día y no usar enjuague o hilo dental, como el principal factor de riesgo de la aparición de caries, ya que todos los pacientes que afirmaron no haber sufrido antes de esta enfermedad, fueron diagnosticados en la consulta odontológica analizada en este estudio.

Asimismo, la prevalencia de dientes perdidos en el grupo B y C, reflejan el resultado de malos hábitos de higiene, consumo de alimentos azucarados y la no visita a consulta odontológica para prevenir las enfermedades. Esto también se evidencia pues en
Periodo. Julio - Diciembre 2021

Vol. 5, Nro. 2, Publicado: 2021-12-31

estos dos grupos es donde se encuentran los diagnósticos de extracciones.

Se pudo concluir la relación entre la caries dental y la ausencia de visita a servicios de salud por parte de los pacientes. Además, al estudiar indirectamente las condiciones socioeconómicas de la población a partir de la interpretación de la actividad económica, se evidencia un mayor número de pacientes relacionados con actividades agrícolas, sin antecedentes de enfermedades personales y/o familiares, que visitan únicamente la Unidad de Salud en casos de enfermedad visible y que por su complejidad deben ser enviados a otros hospitales del sistema de salud del Instituto Ecuatoriano de Seguridad Social.

Se considera que, si la población aplica los métodos de prevención efectivos, la prevalencia de esta enfermedad desciende, de la misma manera que el valor de las mediaciones. Es el caso del grupo A, que asiste con mayor frecuencia a la consulta odontológica a realizarse limpiezas dentales; sin embargo, tiene un mayor porcentaje de diagnóstico de caries, esto debido al escaso conocimiento de los diversos factores que crean el ambiente propicio para la evolución de esta enfermedad. La necesidad se establece entonces, en prevenir la enfermedad en primera instancia; o en su defecto diagnosticarla a tiempo y tratarla correctamente para evitar la pérdida del diente afectado.

\section{Referencias bibliográficas}

1. Ortega Pérez F, Guerrero A, Aliaga P. Determinantes sociales y prevalencia de la caries dental en población escolar de zonas rurales y urbanas de Ecuador. OdontoInvestigación [Internet]. 19 de octubre de 2018 [citado 14 de febrero de 2021];4(2). Disponible en: https://revistas.usfq.edu.ec/index.php/odontoinvestigacion/article/view/1281 2. Ministerio de Salud Pública. Caries. Guías de Práctica Clínica [Internet]. Ministerio de Salud Pública, Dirección Nacional de Normatización-MSP; $2015 . \quad$ Disponible en: https://aplicaciones.msp.gob.ec/salud/archivosdigitales/documentosDirecciones/dnn/archivos/GPC\%20Caries\%20 final\%20\%2024-12-2014.pdf

3. Núñez DP, García Bacallao L. Bioquímica de la caries dental. Revista Habanera de Ciencias Médicas. junio de 2010;9(2):156-66. 
4. Cerrato M, Canales D, Guevara B, Chirinos S, Ramirez R, Espinal T. Frecuencia de caries dentales en pacientes de la clínica odontológica de la Universidad Tecnológica Centroamericana. Innovare: Revista de ciencia y tecnología. 18 de julio de 2019;8(1):18-26.

5. OMS | La OMS publica un nuevo informe sobre el problema mundial de las enfermedades bucodentales [Internet]. WHO. World Health Organization; [citado 14 de febrero de 2021]. Disponible en: https://www.who.int/mediacentre/news/releases/2004/pr15/es/

6. Longbottom CL, Huysmans M-C, Pitts NB, Fontana M. Glossary of Key Terms. Detection, Assessment, Diagnosis and Monitoring of Caries. 2009;21:209-16.

7. Zerón A. Caries dental y caries de hueso. Rev ADM. 25 de junio de 2019;76(3):128-9.

8. Sánchez-Pérez L, Sáenz-Martínez LP, Molina-Frechero N, Irigoyen-Camacho ME, Alfaro-Moctezuma P. Riesgo a caries. Diagnóstico y sugerencias de tratamiento. Rev ADM. 2 de enero de 2019;75(6):340-9.

9. Soria-Hernández MA. Pasado y presente de la caries dental. Acta Pediatr Mex. 2010;31(5):196.

10. Cerón-Bastidas XA. El sistema ICDAS como método complementario para el diagnóstico de caries dental. $2015 ;(2): 10$

11. Olmos P, Piovesan S, Musto M, Lorenzo S, Álvarez R, Massa F. Caries dental. La enfermedad oral más prevalente: Primer Estudio poblacional en jóvenes y adultos uruguayos del interior del país. Odontoestomatología. junio de 2013;15(SPE):26-34.

12. Organización Mundial de la Salud. Salud bucodental: plan de acción para la promoción y la prevención integrada de la morbilidad $2007 . \quad$ Disponible en: https://apps.who.int/iris/bitstream/handle/10665/25842/A60_R17-

sp.pdf;jsessionid=2F6F62E0DE9F4046094638884AD2FE19?sequence=1

13. Cubero Santos A, Lorido Cano I, González Huéscar A, Ferrer García MÁ, Zapata Carrasco MD, Ambel Sánchez JL, et al. Prevalencia de caries dental en escolares de educación infantil de una zona de salud con nivel socioeconómico bajo. Pediatría Atención Primaria. junio de 2019;21(82):e47-59.

14. Aguilar Orozco NY, Navarrete Ayon KB, Martin RRD, Aguilar Orozco SH, Rojas Garcia AR. Dientes sanos, cariados, perdidos y obturados en los estudiantes de la Unidad Académica de Odontología de la Universidad Autónoma de Nayarit. http://www.odontologia.uady.mx/revistas/rol/pdf/V01N2p27.pdf [Internet]. 2009 [citado 20 de febrero de 2021]; Disponible en: http://dspace.uan.mx:8080/xmlui/handle/123456789/565

15. Bueno-Alegría JA, Gutiérrez-Rojo JF, Guerrero-Castellón MP, García-Rivera RN. Índice CPOD y ceo-d de estudiantes de una escuela primaria de la ciudad de Tepic, Nayarit [Internet]. 2019 [citado 20 de febrero de 2021]. Disponible en: https://www.ortodoncia.ws/publicaciones/2019/art-17/

16. Vélez-Vásquez VA, Villavicencio-Caparó E, Cevallos-Romero S, Del Castillo-López C. Impacto de la experiencia de caries en la calidad de vida relacionada a la salud bucal; Machángara, Ecuador. Revista Estomatológica Herediana. julio de 2019;29(3):203-12.

17. González Ferrer V, Alegret Rodríguez M, Martínez Abreu J, González Ferrer Y. Índice de riesgo de caries dental. Revista Cubana de Estomatología. marzo de 2017;54(1):34-47.

18. Andrade Páez M, De la Cruz Cardoso D. Indicadores de prevalencia y de predicción de riesgo de caries dental. Rev Esp Cienc Salud. 15 de junio de 2014;17(1):61-72.

19. González Sanz ÁM, González Nieto BA, González Nieto E. Salud dental: relación entre la caries dental y el consumo de alimentos. Nutrición Hospitalaria. julio de 2013;28:64-71. 
20. Gumila Jardines M, Cuenca Garcell K, Soto Cortés AD, Pérez Bondar V, Del Río LR. Diagnóstico terapéutico para la atención de pacientes con caries dental. Rev Cub Med Mil. 2019;48(2):259-72.

21. Pizarro Merma E. Sistema Internacional de Detección y Valoración de caries dental ICDAS. 2019;5:8.

22. Diaz-Reissner CV, Casas-García I, Roldán-Merino J. Calidad de Vida Relacionada con Salud Oral: Impacto de Diversas Situaciones Clínicas Odontológicas y Factores Socio-Demográficos. Revisión de la Literatura. International journal of odontostomatology. abril de 2017;11(1):31-9.

23. Cueto Rostom V. Diagnóstico y tratamiento de lesiones cariosas incipientes en caras oclusales. Odontoestomatología. noviembre de 2009;11(13):4-15.

24. Mafla AC, Patiño MA. Diferencias de sexo en severidad y factores de riesgo para caries dental y enfermedad periodontal. Revista Nacional de Odontología [Internet]. 22 de abril de 2019 [citado 14 de febrero de 2021];15(28). Disponible en: https://revistas.ucc.edu.co/index.php/od/article/view/2596

25. Toledo Reyes L, Rodríguez Pérez L, Ramos Hurtado I. Riesgo de caries en los niños de tercer grado de la escuela "Fernando Cuesta Piloto". Revista Cubana de Estomatología. septiembre de 2007;44(3):0-0.

26. Cantillo García DAE, Elías Sierra DN, Rodríguez Garbey LY. Nivel de conocimiento sobre caries dental en adolescentes y sus padres. Revista Información Científica [Internet]. 2011 [citado 20 de febrero de 2021];70(2). Disponible en: http://www.revinfcientifica.sld.cu/index.php/ric/article/view/593

27. Gómez Clavel JF, Peña Castillo RE. La valoración del riesgo asociado a caries. Revista ADM. 2014;5865 .

28. Guachisaca Tapia EL, Pérez Paredes ED. Prevalencia de caries dental y su relación con dieta e higiene bucal en pacientes de 18 a 20 años de edad [Internet]. Quito: UCE; 2018 [citado 20 de febrero de 2021]. Disponible en: http://www.dspace.uce.edu.ec/handle/25000/17333

29. Duque de Estrada Riverón J, Hidalgo-Gato Fuentes I, Pérez Quiñónez JA. Técnicas actuales utilizadas en el tratamiento de la caries dental. Revista Cubana de Estomatología. junio de 2006;43(2):0-0.

30. Pallo Guashca RC. Manejo odontológico con el criterio Icdas II, para el diagnóstico de lesiones cariosas tempranas en la unidad de atención odontológica Uniandes [Internet]. 2017 [citado 20 de febrero de 2021]. Disponible en: http://localhost:8080/xmlui/handle/123456789/7305

31. Mayor Hernández F, Pérez Quiñones JA, Cid Rodríguez M del C, Martínez Brito I, Martínez Abreu J, Moure Ibarra MD. La caries dental y su interrelación con algunos factores sociales. Revista Médica Electrónica. junio de 2014;36(3):339-49.

32. Martínez Abreu J, Capote Femenias J, Bermúdez Ferrer G, Martínez García Y. Determinantes sociales del estado de salud oral en el contexto actual. MediSur. agosto de 2014;12(4):562-9. 